\title{
Chapter 1 \\ Building Sustainable Networks: \\ Introducing the Pan Institution Network \\ for Global Health
}

\author{
Margaret S. Winchester, Caprice Knapp, and Rhonda BeLue
}

\subsection{Global Health Collaboration}

In the era of Sustainable Development Goals, multinational non-governmental organizations (NGOs), grand challenges, and record numbers of students seeking educational opportunities abroad, university global health partnerships are quickly becoming a mainstay (Merson 2014). These partnerships can bring together researchers, students, and community members in ways that address education, research, and community health needs. There are compelling advantages to having institutional partnerships so that individuals can collaborate to have a sustainable impact compared to working individually within institutions (Dockrell 2010). A collective base of expertise may also leverage scarce resources and help to develop creative solutions to intractable issues (Binagwaho et al. 2013). Frequently though, universities working together on these issues can reinforce existing disparities and unequal relationships that prioritize the flow of information, bodies, and agendas from higher to lower resourced parts of the globe (Crane 2010; Sewankambo et al. 2015; Syed et al. 2013).

We take as a starting point that partnerships, if built, maintained, and managed in an equitable fashion have the potential to generate a lasting positive impact on global health (Chu et al. 2014; Morse 2014; Pratt and Hyder 2016, 2017). The concept of reverse and global innovation flow is central to this equity, and prioritizes the knowledge and expertise from what is frequently referred to as the Global South (Syed et al. 2013; Crisp 2014). Reverse and global innovation flows create possibilities for relevant community-based research, open access dialogue, and

M. S. Winchester $(\square) \cdot$ C. Knapp

Health Policy and Administration, Pennsylvania State University, University Park, PA, USA

e-mail: winchester@psu.edu

R. BeLue

Health Management and Policy, St. Louis University, St. Louis, MO, USA 
reciprocity and respect (Binagwaho et al. 2013; Crisp 2014; Snowdon et al. 2015). At a fundamental level, this is a challenge to the traditional dynamics of NorthSouth models of collaboration. The global flow of ideas in the realm of health is by no means new, though typically only tacitly acknowledged when flowing in "reverse."

Given the ever-present community-academic and North-South imbalances, the Pan Institution Network for Global Health (PINGH) employs a participatory governance and research approach to bring a balance of power by featuring local stakeholders as content and context experts. First, PINGH has a rotating governance structure. Each university partner has a champion who represents his/her institution for all PINGH activities. Second, leadership rotates on a three-year cycle. Each institution takes turns leading PINGH initiatives as well as hosting the annual PINGH collaborative meeting, in order to offer an opportunity for all PINGH members to experience and gain insight on the institutional and local cultures of each PINGH member and to defray costs of hosting the meeting for any one institution. These activities also insure participation and contribution from all PINGH members in the global North and South.

To promote inclusivity, we invite community stakeholders to our annual meetings to ensure that PINGH objectives are relevant to the local communities in which PINGH institutional members serve. Local NGOs, government policy makers, and other local stakeholders have attended PINGH meetings to help our partners reflect and strategize on how global health research and capacity building can be translated to local policy and practice. Our approach to community stakeholder involvement is discussed in further detail in this chapter.

\subsection{History of PINGH}

The Pan Institution Network for Global Health (PINGH) is the result of a targeted interest in using university partnerships to address global health needs (Winchester et al. 2016). In 2014, Pennsylvania State University hosted a meeting to discuss potential mechanisms and collaborations among more than a dozen global universities. After the meeting, faculty members across the universities established basic guidelines for working together, including a steering committee of 'champions' from each institution and two thematic priorities within the broader field of global health (detailed below). Following a consolidation of interest, the core membership of PINGH included six universities: Albert Ludwigs University of Freiburg (Germany), Savitribai Phule Pune University (India), The University of the West Indies (Barbados, Jamaica, and Trinidad and Tobago), University of Cape Town (South Africa), University of Limpopo (South Africa), and Pennsylvania State University. More recently, Mountcrest University (Ghana) has also joined the group.

Central to PINGH's vision are three pillars of collaboration: (1) research, (2) education, and (3) capacity building. Each of these pillars is dependent on the others

${ }^{1}$ Formerly the Pan University Network for Global Health (PUNGH). 
and none represents the sole reason for the network. PINGH is still relatively young, but has a growing portfolio of activities within each area.

To encourage collaboration, PINGH leadership initiated several activities, including pilot projects. The pilot projects were intended to serve a dual purpose: First to encourage collaboration between PINGH members. Pilot project funding guidelines required that at least two PINGH members were included and at least one global South institution was included on every funded project. Secondly, pilot projects were selected to advance PINGH pillars. During the first two years after the network's founding, through funding from Pennsylvania State University, PINGH was able to offer two rounds of seed grants, totaling \$100,000 USD. We funded 11 projects, described below. Many of these projects are also further detailed in subsequent chapters, including successes, challenges, and lessons learned.

Several grants were awarded in early 2014: three capacity building meetings, two secondary data analyses, and two primary research studies. One project organized a conference to establish a multidiscplinary network of emerging scholars on migration, urbanization and health in Southern Africa and included partners from Pennsylvania State University, University of Cape Town, and University of Witwatersrand. The group hosted a workshop in July 2015 that included three keynote presentations and ten presentations from early career researchers across Southern Africa. Pre- and post-workshop, emerging researchers were paired up with established scholars to exchange comments on drafts of papers, feedback on presentations, and even grant and publication opportunities. Another meeting was organized in Jamaica in April 2015 to facilitate writing and partnerships between investigators at Pennsylvania State University, the University of the West Indies, and University of Cape Town, and focused on the intersection of HIV and chronic diseases. The group completed a systematic review and have remained partners, submitting multiple grants together, and eventually combined with another project focused on improving systems for chronic care. The group studying the intersection of chronic and infectious diseases in health systems included partners from Pennsylvania State University, University of Limpopo, and University of Cape Town. They collected primary data, developed an optimization model for care, completed the training of two graduate students in public health, and have published one paper to date (Oni et al. 2014). Using existing data sets, another project examined the relationship between body mass index and mortality, with investigators from Pennsylvania State University, Savitribai Phule Pune University, Hebei Union University, and Kailuan Hospital. This project resulted in two peer-reviewed publications, but the investigators decided not to pursue an ongoing partnership (Cheng et al. 2016a, b). The other project using secondary data identified priority areas for mother and child interventions in Cape Town, with partners from Pennsylvania State University, University of Cape Town, University of Freiburg, and Savitribai Phule Pune University. As a result, the investigators have had ongoing partnerships with multiple grants submitted and one published paper to date (Mumm et al. 2017). One final project set out to develop new partnerships between Pennsylvania State University, Savitribai Phule Pune University, and the University of Cape Town to study vitamin D deficiency and pregnancy. This grant led to 
multiple meetings, but the group ultimately was unable to proceed with data collection for a variety of reasons.

In January 2016, a second round of five grants was funded. This round focused on scaling up existing research collaborations and building capacity among junior network members. These projects all required some sort of matching funds or resources in kind from partner institutions. The team that investigated the intersection of HIV and chronic diseases was funded a second time to study systems for strengthening chronic care in South Africa and the Caribbean, with partners from Pennsylvania State University, the University of the West Indies, University of Cape Town, and University of Limpopo. As a direct result of this project, two additional master of public health students were trained and several article manuscripts are in process. The group has identified external funding and will use the data collected as a pilot for this larger project to improve healthcare for multiple morbidities. Another research project with researchers at Pennsylvania State University and Savitribai Phule Pune University has been studying the healthcare access of informal workers in India and the United States. This group has collected data in New York, a student analyzed the data for a master's thesis in Health Policy and Administration, and the group is in the process of working with community partners to determine next steps. The 'Secret History' methodology is a South African-developed training for empathic healthcare; a group from University of Cape Town, University of Freiburg, and Pennsylvania State University was funded to bring this training to healthcare workers in Germany. The group conducted two sets of trainings, and then disseminated the information and additional training among PINGH members at one of the annual network meetings. Three papers from the trainings are in process, to detail the adaptation of the method across settings. Another group hosted a workshop on urbanization and health for young scholars and graduate students in Pune, India in early 2017. Attended by more than two dozen faculty, the workshop facilitated proposal writing and other urban health activities at Savitribai Phule Pune University. Finally, a small grant was given to faculty from Pennsylvania State University and Savitribai Phule Pune University to explore the development of online modules in public health and health systems. The group faced significant logistical difficulties in developing a formal course, but ended up collaborating in different ways, including the hosting of international public health students in India and reciprocal review of materials.

Some of these projects have blossomed into further ongoing partnerships and activities. PINGH members meet in person annually, in addition to regular electronic and video communication for projects and between champions. PINGH also encourages bilateral and smaller group collaborations. Global North-South bilateral relationships and projects are especially encouraged. Each of the member institutions has branched out to develop these relationships outside the network.

PINGH facilitates the development of global health infrastructure within each member institution. PINGH members are encouraged to strengthen global health activities within their own institutions and then share these strategies and activities with other PINGH members at annual meetings. Global health seminar series, 
global health policy workshops, and events offered at individual PINGH institutions are advertised through the PINGH newsletter and emails so that partner institutions may replicate in their own institutions. Bilateral relationships have included research projects, education and training initiatives, and student development such as participation as a reviewer, examiner or committee member on graduate student thesis and dissertation committees.

\subsection{Priority Areas}

Within the ever-expanding field of global health, PINGH has selected two specific priority areas: multiple morbidities and urban health. Both of these areas are in line with the shifting global burden of disease and growing population (Winchester et al. 2016). In today's globalized world, many low- and middle-income countries are undergoing rapid changes that are conducive to both ongoing infectious disease burdens and growing rates of noncommunicable diseases (Murray and Lopez 2013). In particular, rapid urbanization, mechanization of the rural economy, and the increasing activities of transnational food, drink, and tobacco corporations are all associated with behavioral changes that increase the risk of noncommunicable diseases. As a result, population health profiles and patterns are rapidly changing with an increase in cardiovascular and metabolic disorders. Population estimates suggest that by 2045 there will be over six billion urban residents, out of a global population of nine billion. The global, regional, and local variation in the trends we see today relating to shifting demographics and spatial inequalities ensure that the majority of population growth will occur in developing countries, with relative growth being highest across Africa. While megacities (the very largest global metropolises) are often highlighted, urban growth has occurred across the entire settlement system, reinforcing existing health challenges as well as generating new ones (Montgomery 2008). Processes of urbanization provide the dynamic backdrop to how we conceptualize and define global health challenges.

\subsection{Framework and Guiding Principles}

Guiding all of PINGH's activities and selection of priorities has been a commitment to equity, both in collaboration and global health more broadly. These two aims are deeply entwined and guide our governance practices within the network. We outline our logic model elsewhere (Winchester et al. 2016), but note that it is built on two assumptions: (1) that partnerships and the reciprocal flow of innovation is necessary to address global health and health care challenges, and (2) collaboration requires engaged network members, collective decision making and open communication. Building on Pratt and Hyder's (2017) framework for governance of global health consortia, we aim for a deliberate and inclusive process among our partners. They 
emphasize the two aspects of shared sovereignty and shared resources, as the foundation of equitable partnerships. While PINGH's founding was initiated by one US-based university, we have actively sought to include representatives from each institution in a single governing board. Partners have nominated a 'champion' for their university, and this person is part of the network's governing board. Champions are chosen for their standing within the institution, ability to connect interested faculty, and to potentially garner resources. The board has one director, currently at Pennsylvania State University, where there is also a full time faculty coordinator for managing network activities. In order to maintain equity among members, we are moving to a rotating model, which will allow each institution to take up to a threeyear term 'hosting' the network, directorship, and coordination. Not all partners have access to similar levels of resources at their institutions, and may opt for a shorter term.

In keeping with our goal to conduct research that can be readily translated to policy, advocacy, and practice, we include community partners, practitioners, and government representatives at all of our meetings. We follow the Health in All Policies (HiAP) approach (WHO 2014), which posits that policies made in all sectors can significantly affect population health and health equity. In a global and interconnected society, health is influenced by demographic, environmental, and social forces. In the spirit of the HiAP framework, at our annual meetings and all PINGH sponsored events, we include not only local stakeholders that represent health care organizations, but stakeholders who represent social, environmental, transportation, and economic sectors that affect health through social processes. Inviting a diverse group of local stakeholders also offers an opportunity for PINGH colleagues from other countries to more deeply understand the sociopolitical dynamics of their collaborators' countries which facilitates improved and more informed research collaborations.

\subsection{Outline of Chapters and Volume}

Each chapter in this edited volume showcases one project or aspect of a project completed by PINGH members. While there are many intersections and overlaps among the projects and authors, we have divided up the sections to focus on lessons learned through specific activities. We are the first to admit that PINGH is still a young network and we are still learning. The chapters each focus on lessons learned through trial and error. While some groups have been able to establish best practices, others share the challenges and pitfalls that can happen when working across contexts and how to avoid replicating our mistakes.

This book consists of two sections that provide case studies, evidence, and experiences related to essential elements of the PINGH model of cross-national partnerships including education, capacity building, and research. The first section focuses specifically on the PINGH education and capacity building pillars. Chapters focus on strategies and lessons learned related to global health education for diverse stu- 
dents, the process of building collaborative research capacity to effectively study the effects of urbanization, and the cultural and contextual adaptation of women's health care training protocols from the global south to the global north. The final section of the Education and Collaboration section discusses strategies to sustain and finance global health collaboration.

The second section entitled Research Lessons consists of case studies and lessons learned from cross-national research initiatives that were initiated through PINGH pilot project funding. These chapters present work directly related to our research foci: multiple morbidity and urbanization. All case studies approach research from a cross-national perspective. The first chapter of this section, Chap. 5, explores the concept of patient workload in relation to managing multiple chronic illnesses, specifically HIV and Diabetes. Chapters 6 and 7 explore urbanization from two points of view. First is a cross-national study on challenges and needs of informal wastepickers in urban context. Second, is the application of a maternalchild health research framework for identifying maternal child health resources in urban areas, and how this framework can be applied to broad urban settings. The concluding chapter discusses ways forward and long-term needs to advance global health research and collaborations. Lastly, this edited volume ends with a personal reflection from a long time global health scholar and the founder of the PINGH network.

\section{References}

Binagwaho A, Nutt CT, Mutabazi V, Karema C et al (2013) Shared learning in an interconnected world: innovations to advance global health equity. Global Health 9(1):37. doi. org/10.1186/1744-8603-9-37

Cheng FW, Gao X, Mitchell DC et al (2016a) Body mass index and all-cause mortality among older adults. Obesity 24(10):2232-2239. https://doi.org/10.1002/oby.21612

Cheng FW, Gao X, Mitchell DC et al (2016b) Metabolic health status and the obesity paradox in older adults. J Nutr Gerontol Geriatr 35(3):161-176. https://doi.org/10.1080/21551197.2016. 1199004

Chu KM, Jayaraman S, Kyamanywa P et al (2014) Building research capacity in Africa: equity and global health collaborations. PLOS Med 11(3):e1001612. doi.org/10.1371/journal. pmed. 1001612

Crane JT (2010) Unequal 'partners.' AIDS, academia, and the rise of global health. Behemoth-a Journal on Civilisation 3(3):78-97. doi.org/10.6094/behemoth.2010.3.3.685

Crisp N (2014) Mutual learning and reverse innovation-where next? Global Health 10(1):14. doi. org/10.1186/1744-8603-10-14

Dockrell HM (2010) Presidential address: the role of research networks in tackling major challenges in international health. Int Health 2(3):181-185. doi.org/10.1016/j.inhe.2010.07.004

Merson MH (2014) University engagement in global health. N Engl J Med 370(18):1676. https:// doi.org/10.1056/NEJMp1401124

Montgomery MR (2008) The urban transformation of the developing world. Science 319(5864):761-764. https://doi.org/10.1126/science.1153012

Morse M (2014) Responsible global health engagement: A road map to equity for academic partnerships. J Grad Med Educ 6(2):347-348. doi.org/10.4300/JGME-D-14-00165.1 
Mumm R, Diaz-Monsalve S, Hänselmann E et al (2017) Exploring urban health in Cape Town, South Africa: an interdisciplinary analysis of secondary data. Pathog Glob Health 111(1):7-22. dx.doi.org/10.1080/20477724.2016.1275463

Murray CJ, Lopez AD (2013) Measuring the global burden of disease. N Engl J Med, 369(5):448-457

Oni T, McGrath N, BeLue R et al (2014) Chronic diseases and multi-morbidity-a conceptual modification to the WHO ICCC model for countries in health transition. BMC Public Health 14(1):575. doi.org/10.1186/1471-2458-14-575

Pratt B, Hyder AA (2016) Governance of transnational global health research consortia and health equity. Am J Bioeth 16(10):29-45. dx.doi.org/10.1080/15265161.2016.1214304

Pratt B, Hyder AA (2017) Governance of global health research consortia: sharing sovereignty and resources within future health systems. Soc Sci Med 174:113-121. doi.org/10.1016/j. socscimed.2016.11.039

Sewankambo N, Tumwine JK, Tomson G et al (2015) Enabling dynamic partnerships through joint degrees between low- and high-income countries for capacity development in global health research: experience from the Karolinska Institutet/Makerere University partnership. PLOS Med 12(2):e1001784. doi.org/10.1371/journal.pmed.1001784

Snowdon AW, Bassi H, Scarffe AD et al (2015) Reverse innovation: an opportunity for strengthening health systems. Global Health 11(1):2. doi.org/10.1186/s12992-015-0088-x

Syed SB, Dadwal V, Martin G (2013) Reverse innovation in global health systems: towards global innovation flow. Global Health 9(1):36. doi.org/10.1186/1744-8603-9-36

Winchester MS, BeLue R, Oni T et al (2016) The Pan-University Network for Global Health: framework for collaboration and review of global health needs. Global Health 12(1):13. doi. org/10.1186/s12992-016-0151-2

World Health Organization (2014) Health in all policies: Helsinki statement. Framework for country action. http://apps.who.int/iris/bitstream/10665/112636/1/9789241506908_eng.pdf. Accessed 30 Oct 2017

Open Access This chapter is licensed under the terms of the Creative Commons Attribution 4.0 International License (http://creativecommons.org/licenses/by/4.0/), which permits use, sharing, adaptation, distribution and reproduction in any medium or format, as long as you give appropriate credit to the original author(s) and the source, provide a link to the Creative Commons license and indicate if changes were made.

The images or other third party material in this chapter are included in the chapter's Creative Commons license, unless indicated otherwise in a credit line to the material. If material is not included in the chapter's Creative Commons license and your intended use is not permitted by statutory regulation or exceeds the permitted use, you will need to obtain permission directly from the copyright holder.

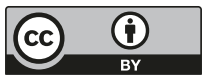

